\title{
Ischaemic lesions of the alimentary tract
}

\author{
G. S. A. McDONALD AND D. O'B. HOURIHANE \\ From the Central Histopathology Laboratory, School of Pathology, Trinity College, Dublin
}

SYNOPSIS The clinical and pathological findings in 23 patients with ischaemic lesions of the alimentary tract (ischaemic enterocolitis) are described. These are compared with findings in 13 patients with occlusive thrombosis of the superior mesenteric artery. The pathological features distinguishing the two conditions are discussed.

Ischaemic enterocolitis was found to be a relatively common condition ( $0.6 \%$ of necropsies). The occurrence of mucosal fibrosis (a hitherto undescribed feature) has been of help in the biopsy diagnosis of ischaemic lesions of the gut.

Ischaemic enterocolitis is total or partial infarction of the intestine in the absence of occlusion of a major mesenteric blood vessel (McGovern and Goulston, 1965; Marston, Pheils, Thomas, and Morson, 1966). Since the first description of the condition (Moulonguet, 1931) its postoperative occurrence (Penner and Bernheim, 1939) and its frequent association with cardiovascular disease (Wilson and Qualheim, 1954; Musa, 1965) have been emphasized. Hypotension precedes the onset of infarction in a proportion of cases (McGovern and Goulston, 1965; Ming, 1965). Patients with ischaemic enterocolitis are usually elderly and present with abdominal pain, diarrhoea, and the passage of blood per rectum. The pathological lesions range in severity from mucosal haemorrhage to gangrene of the bowel. Healing takes place by granulation tissue and fibrosis in a manner reminiscent of a healing myocardial infarct (Marston et al, 1966; Morson, 1966,1968 ), and strictures of the bowel may result. The main distinction between ischaemic enterocolitis and infarction due to superior mesenteric artery thrombosis is the absence of occlusion of a major mesenteric artery in the former.

The numerous publications describing the pathological findings in ischaemic enterocolitis have clarified the diagnostic criteria, both clinical and pathological. In the case of pathological material this is especially true where actual infarction with necrosis can be demonstrated. Where only fibrosis is present, however (for example in a stricture), the demonstration of ischaemia as the underlying cause may be difficult. The present paper describes a pathological finding which may be of help in the diagnosis of healed ischaemic lesions of the intestine. Received for publication 2 June 1971.
In addition the findings in 23 patients with ischaemic enterocolitis and in 13 patients with superior mesenteric artery thrombosis are described and compared.

\section{Material}

Pathological specimens received in the School of Pathology, Trinity College, Dublin, were examined. They were obtained from surgical operations and necropsies performed in the Federated Dublin Voluntary Hospitals. The specimens consisted of segments of intestine or intestinal biopsies from patients admitted to hospital during the years 1965 to September 1970. Clinical histories were obtained from hospital records. The presence or absence of cardiovascular disease in each patient was established where possible from clinical notes and necropsy protocols.

Twenty-seven controls were selected at random from surgical specimens received during the same period of time. These consisted of patients with adenocarcinoma of the colon (11), adenocarcinoma (primary and secondary) of the small intestine (2), ileocaecal tuberculosis (1), Crohn's disease of the small intestine and colon (8), non-specific ileitis (1), and ulcerative colitis (4). Their average age was 54 years (range 22 to 89 years) and there were 15 males and 12 females.

\section{Results}

There were 23 patients with non-occlusive ischaemic enterocolitis and 13 patients with thrombosis of the superior mesenteric artery. The incidence at necropsy and clinical findings are summarized in Table $\mathbf{I}$. 
During the period under study, 2080 necropsies were performed of which 13 were on patients with ischaemic enterocolitis and 13 on patients with superior mesenteric artery thrombosis. This is an incidence at necropsy of $0.6 \%$ for each condition. There was a slight preponderance of females in both conditions but the average ages were comparable66.1 years for patients with ischaemic enterocolitis and 66.6 years for patients with superior mesenteric artery thrombosis.

\begin{tabular}{lll}
\hline & \multicolumn{2}{l}{ No. of Patients } \\
\cline { 2 - 3 } & $\begin{array}{ll}\text { Ischaemic } \\
\text { Enterocolitis }\end{array}$ & $\begin{array}{l}\text { Superior Mesenteric } \\
\text { Artery Thrombosis }\end{array}$ \\
\hline Total number of patients & 23 & 13 \\
Necropsy incidence (\%) & $0 \cdot 6$ & $0 \cdot 6$ \\
Males : female ratio & $10: 13$ & $6: 7$ \\
Age Average & $66 \cdot 1$ & $66 \cdot 6$ \\
(yr) Range & $39-83$ & $56-76$ \\
Clinical features & & \\
Abdominal pain, & & 7 \\
vomiting, diarrhoea & 14 & 0 \\
Blood per rectum & 9 & 3 \\
Hypotension & 6 & 3 \\
None & 2 & \\
\hline
\end{tabular}

Table I Clinical findings

Abdominal pain with or without vomiting and/or diarrhoea were the usual presenting features of patients with ischaemic enterocolitis and patients with superior mesenteric artery thrombosis. Nine patients with the former condition passed bright red blood per rectum but none of the patients with superior mesenteric artery thrombosis showed this feature. A state of 'collapse' with hypotension accompanied or followed the onset of abdominal pain in six patients with ischaemic enterocolitis and in three with superior mesenteric artery thrombosis. Five patients had no symptoms referable to the gastrointestinal tract, the lesions being discovered at necropsy.

\section{Pathological Features}

No occlusion of the superior mesenteric artery was found at necropsy in any patient with ischaemic enterocolitis, although a potential source of embolus was present in three. The mesenteric arteries were found to be normal at operation in two patients. Unfortunately no comment was made in the hospital records about the state of the mesenteric arteries in the remaining eight patients on whom no necropsy was performed.

Table II summarizes the types of lesion found and the sites at which they were located along the gastrointestinal tract. Most of the patients had full-

\begin{tabular}{lcc}
\hline Type and Site of Lesion & $\begin{array}{l}\text { No. of Patients } \\
\text { with Ischaemic } \\
\text { Enterocolitis }\end{array}$ & $\begin{array}{l}\text { No. of Patients } \\
\text { with Superior } \\
\text { Mesenteric Artery } \\
\text { Thrombosis }\end{array}$ \\
\hline Gangrene & 2 & 1 \\
Infarction & 14 & 10 \\
Stricture & 5 & 0 \\
Mucosal atrophy and & 2 & 1 \\
fibrosis & 2 & 0 \\
Stomach & 1 & 13 \\
Small intestine & 17 & 0 \\
Colon & 4 & 0 \\
Rectum & 2 & \\
\hline
\end{tabular}

Table II Multiple sites

thickness or partial thickness infarction with or without gangrene (putrefaction). Two patients had mucosal atrophy and fibrosis. Most of the lesions of ischaemic enterocolitis were distributed in a patchy manner in the small intestine and/or the colon. One patient had ischaemic ulcers involving the stomach, small intestine, and colon, but sparing the rectum.

The rectum was involved in two patients and biopsy showed mucosal atrophy and fibrosis, thought to be due to ischaemia.

The patients with superior mesenteric artery thrombosis had infarction with or without gangrene(putrefaction) but one had only mucosal atrophy and fibrosis. A patient who had only congestion of the intestine is not included in the Table. The lesions, in contrast to those of ischaemic enterocolitis, were all in the distribution of the superior mesenteric artery. In 10 patients most of the small intestine was diffusely involved, in contrast to the patchy distribution of ischaemic enterocolitis.

Gangrene of ischaemic enterocolitis appeared as the typical dull blue/grey discoloration of the intestine with a putrefactive odour. Histologically, full-thickness infarction of the intestine with massive inflammatory reaction was seen (Fig. 1) and numerous Gram-positive bacilli were present in the mucosa of one patient. The mesentery in each case of gangrene showed fresh venous thrombi and in one an organized thrombus in a small artery.

The appearances of full-thickness infarction without gangrene in ischaemic enterocolitis are shown in Figure 2. The discoloration was usually patchy in distribution and well demarcated from the neighbouring unaffected intestine. The mucosa was typically congested and bore numerous small round ulcers up to $1 \mathrm{~cm}$ diameter. In two patients changes were confined to the mucosa, the bowel being normal externally. Bloodstained peritoneal fluid was present in three patients.

Strictures appeared as well demarcated, indurated 


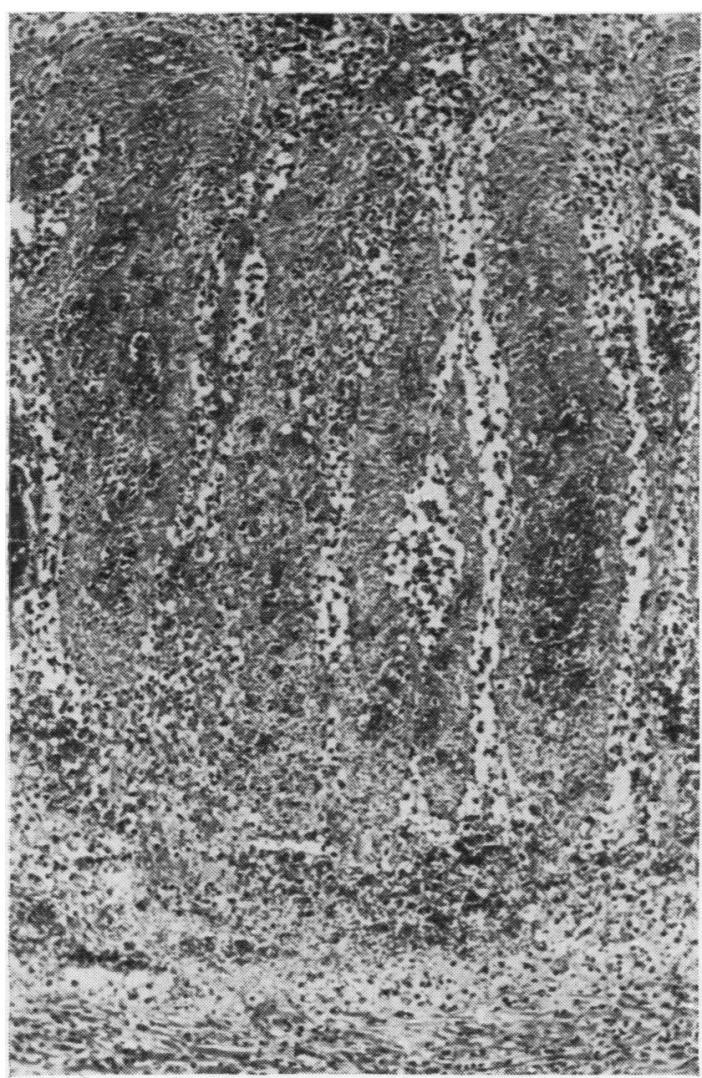

Fig. 1 Haemorrhagic infarction of the muscularis propria in a case of fu!l-thickness ischaemic enterocolitis H. \& E. 63 areas of thickening of the bowel wall with narrowing of the lumen. The mucosa overlying the stricture was ulcerated in two patients. Strictures were located in the sigmoid colon (1), in the descending colon (1), in the ileum (2), and in the jejunum (1). One patient had two strictures in the ileum.

Typical histological appearances of the bowel in cases of infarction and ulceration in the absence of occlusion of a major blood vessel (ischaemic enterocolitis) are shown in Figures 3 and 4. There was coagulative necrosis involving the mucosa only (Fig. 3) in 12 patients and extending to involve the full thickness of the intestinal wall in eight patients. Early lesions appeared as fresh haemorrhage in the mucosa and submucosa. A remarkable finding was infarction of the superficial half of the mucosa (Fig. 4). In the submucosa, characteristic features were the presence of cedema, dilated blood vessels, and thrombosed venules (Fig. 4). An intense inflammatory reaction was present in the region of ulcers with minimal inflammation in other areas. Fibrosis was present in addition to fresh infarction in five patients (other than those with strictures) suggesting previous episodes of ischaemia. Acute arteritis was seen close to ulcers in two patients (Fig. 5). The mesentery contained thrombosis of small veins in two patients.

Patients with strictures showed fibrosis of the submucosa and muscularis propria. All but one had neighbouring recent infarction as well. One patient had no infarction but showed foci of fibrosis in the mucosa in addition to mucosal ulceration and submucosal haemorrhage and thrombosis. This collagen formation in the mucosa is shown in Figure 6. The

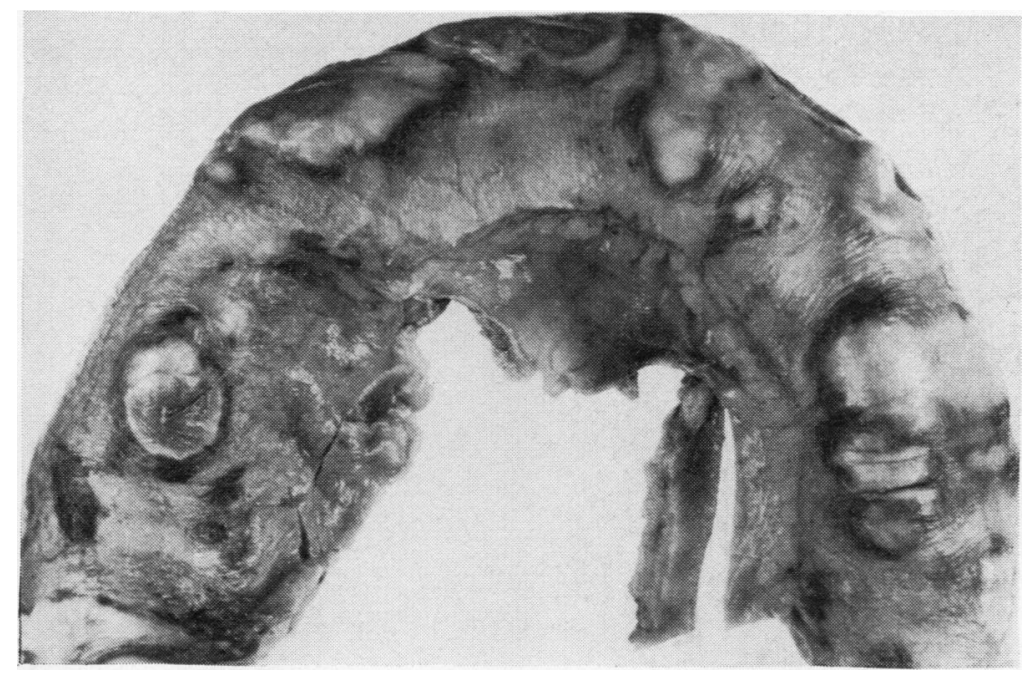

Fig. 2 Multiple discrete infarcts of intestine in ischaemic enterocolitis. Note haemorrhagic borders surrounding pale necrotic zones. 


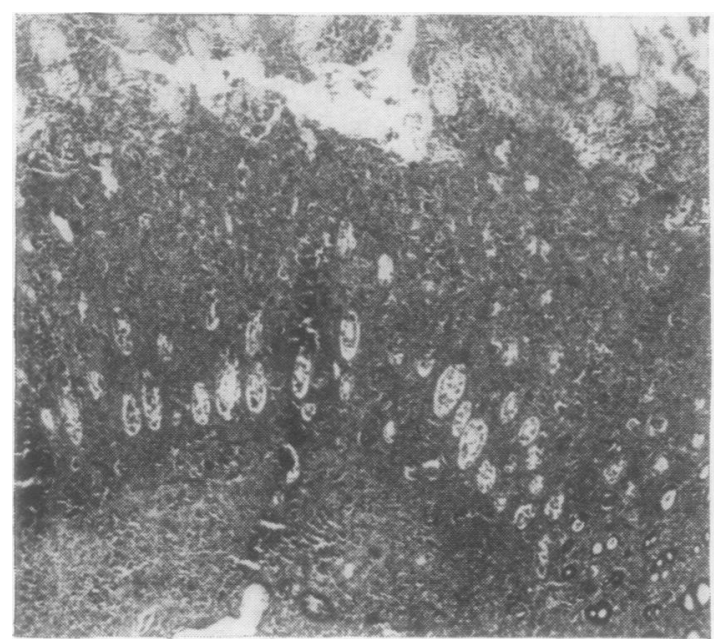

Fig. 3 Haemorrhagic necrosis of mucosa with ulceration and exudate at surface. Viable mucosa in right-hand corner of the photograph. H. \& E. $\times 40$.

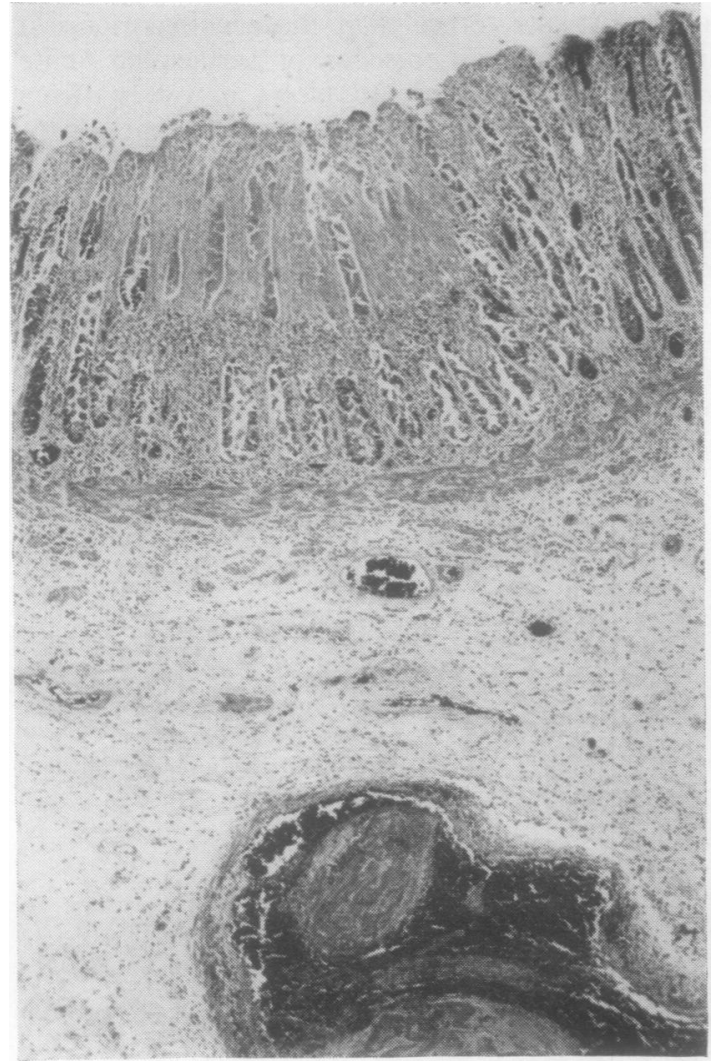

Fig. 4 Coagulative necrosis of superficial half of mucosa in ischaemic enterocolitis. There is an inflammatory reaction separating necrotic from viable mucosa. The submucosa is oedematous, and the vein in the lower part of the photograph contains a thrombus. H. \& E. $\times 25$.

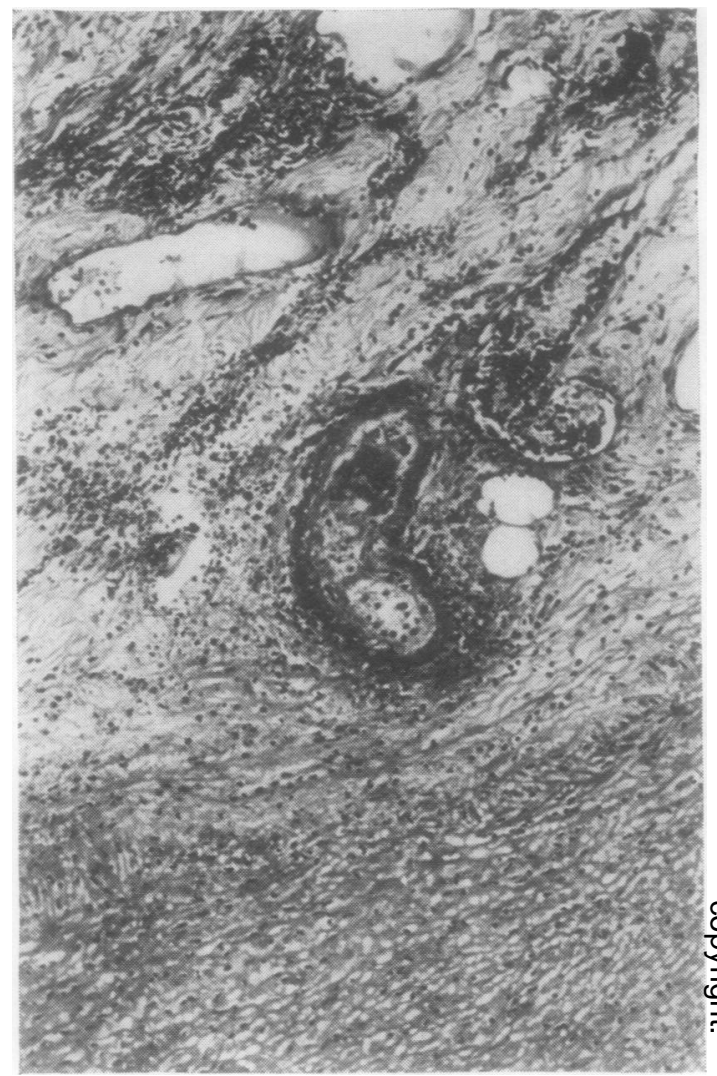

Fig. 5 Fibrinoid necrosis of small artery in ischaemic enterocolitis beside muscularis propria. Small thrombus in lumen. Acute inflammatory reaction surrounds the necrotic vessel. $H . \&$ E. $\times 63$.

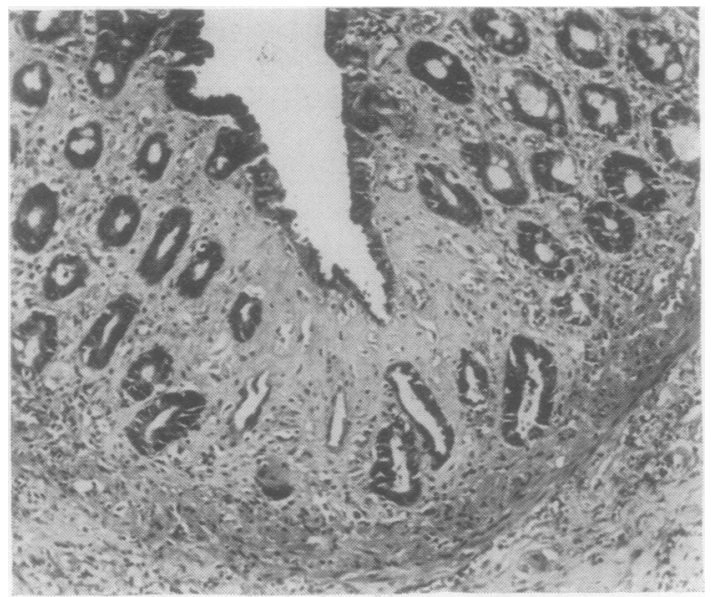

Fig. 6 Fibrosis of lamina propria is shown in centre of photograph, with thinning of mucosa and atrophy of crypts in ischaemic enterocolitis. $H . \& E . \times 63$. 


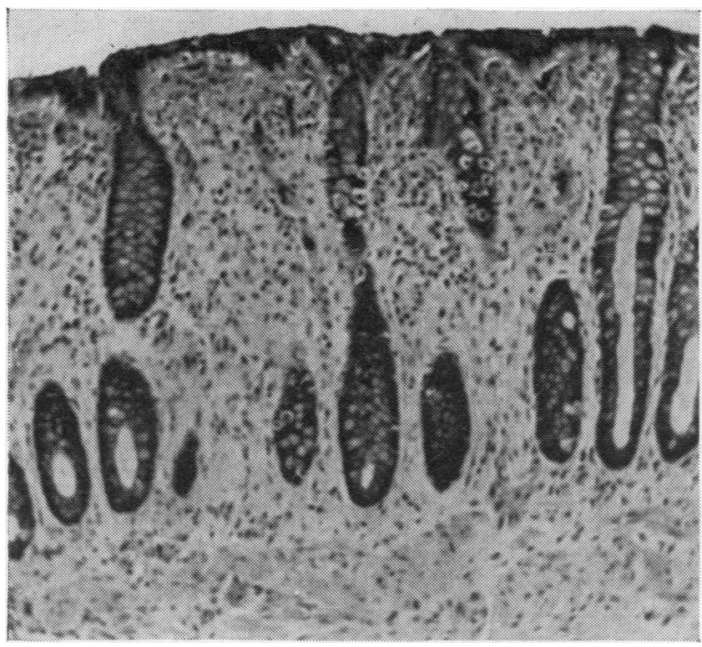

Fig. 7 Separation of crypts in rectal mucosa by pale staining material (amyloid negative) in ischaemic enterocolitis. H. \& E. $\times 63$.

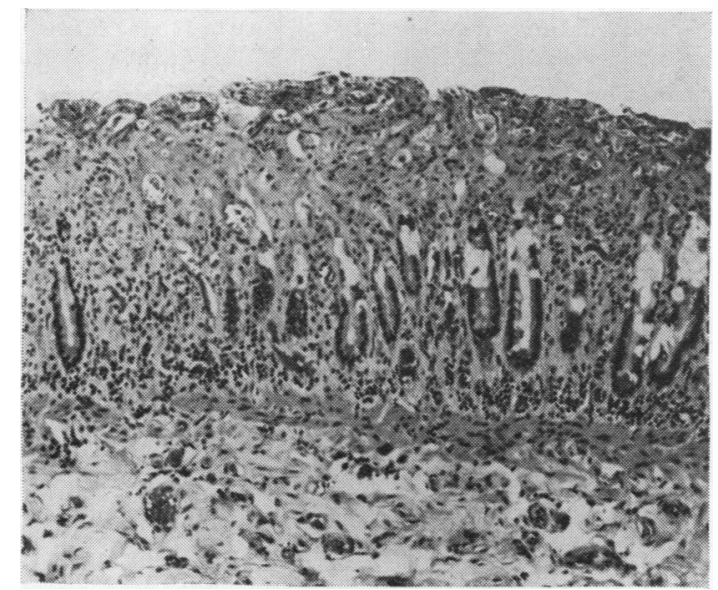

Fig. 8 Jejunal biopsy showing flat atrophic mucosa with fibrosis of its superficial half. From a case of thrombosis of superior mesenteric artery. H. \& E. $\times 63$.

area stained pink (ie, as collagen), with Van Gieson's stain.

Two patients presented some unusual features. Changes appeared to be confined to the rectum and consisted of atrophy of the mucosa with loss of glands and replacement by fibrosis (Fig. 7). Thrombi and sludging of red blood cells were present in the mucosal capillaries of one patient who had multiple myeloma and an abnormal circulating protein. Both patients had passed blood per rectum, and the histological changes were interpreted as those of chronic ischaemia.
The acid ferricyanide stain for haemosiderin was performed on seven specimens but no haemosiderin was detected, nor was brown pigment noted in any section in the remaining 16 cases.

The histological appearances of the intestine in patients with superior mesenteric artery thrombosis were those of infarction. There were two exceptions. One patient already mentioned had only congestion of the intestine. The second had an ischaemic segment of jejunum from which a biopsy was taken. The histological features are shown in Figure 8. The mucosa shows fibrosis and flattening of the villi.

None of the control patients showed coagulative necrosis or submucosal thrombi. No mucosal fibrosis was seen in any patient either in the mucosa affected by the respective lesions nor in the adjacent normal mucosa. Some pink strands of collagen (Van Gieson stain) were seen in the mucosa at the edge of an ulcer in one patient with Crohn's disease but this bore no resemblance to the pink hyaline-like replacement of glands seen in ischaemic colitis (Fig. 6).

\section{ASSOCIATED DISEASE}

The major diseases present in association with ischaemic enterocolitis and superior mesenteric artery thrombosis are summarized in Table III.

\begin{tabular}{lll}
\hline Associated Disease & $\begin{array}{l}\text { No. of Patients } \\
\text { with Ischaemic } \\
\text { Enterocolitis }\end{array}$ & $\begin{array}{l}\text { No. of Patients } \\
\text { with Superior } \\
\text { Mesenteric } \\
\text { Artery Thrombosis }\end{array}$ \\
\hline $\begin{array}{l}\text { Cardiovascular } \\
\begin{array}{l}\text { Pulmonary (chronic } \\
\text { bronchitis and }\end{array}\end{array}$ & 10 & 13 \\
$\begin{array}{c}\text { emphysema) } \\
\text { Debilitation, eg, carcino- } \\
\text { matosis, severe haemor- } \\
\text { rhage from peptic ulcer, }\end{array}$ & 5 & \\
$\begin{array}{c}\text { postoperative state } \\
\text { Not known }\end{array}$ & 7 & 0 \\
Thromboembolism & 6 & 0 \\
\hline
\end{tabular}

Table III Disease associated with ischaemic enterocolitis

Fifteen patients with ischaemic enterocolitis were found to have associated disease. Ten had cardiovascular disease which included ischaemic and hypertensive heart disease. No associated disease was found in two patients. In six patients the presence or absence of associated disease is not known. A potential source of embolization in the form of mural thrombus in the left atrial appendage on the left ventricle of the heart was present in three patients. Other organs were infarcted but no occlusion of a major mesenteric artery was found in any case. 
In contrast to the patients with ischaemic enterocolitis, all patients with superior mesenteric artery thrombosis had cardiovascular disease. In 10 of the 13 patients there was a mural thrombus in the heart with embolization. This difference in the incidence of thromboembolism in the two conditions (Table III) is statistically significant $(P<0 \cdot 01)$, as is the difference in the incidence of cardiovascular disease.

None of the patients in either group was receiving enteric-coated potassium chloride tablets.

\section{PROGNOSIS}

Seven of the 23 patients with ischaemic enterocolitis survived but all 13 patients with superior mesenteric artery thrombosis died. Of these seven survivors, five had strictures or mucosal atrophy and two had infarction without stricture formation. Both patients with gangrene died.

\section{Discussion}

Our results indicate that in Ireland ischaemic enterocolitis occurs with similar frequency to that reported from other countries (Larsen, 1970) although the absence of radiological investigations would suggest that many clinicians are not aware of its symptoms. This failure to consider ischaemic enterocolitis in diagnosis led to many laparotomies, so that we have received a disproportionate number of early lesions compared with those reported elsewhere, and in contrast to other series, we have had no radiological studies.

This series demonstrates the wide spectrum of ischaemic lesions of the intestines, doubtless due to its retrospective nature. The patients with strictures are in the minority, although this type of lesion appears more common in centres where greater emphasis is placed on clinical or radiological diagnosis.

There may be nothing in the clinical features to distinguish ischaemic enterocolitis from the effects of thrombosis of the superior mesenteric artery. Although in our series none of the patients with superior mesenteric artery thrombosis passed blood per rectum, this fact is unusual since both stools and vomit may contain blood in this condition (Wang and Reeves, 1960). A statistically significant difference between the incidence of thromboembolism in the two conditions was found and this has been reported by Larsen (1970). However, it probably reflects the differences in the aetiology of the two conditions, and is not of help in the antemortem diagnosis.

Pathologically, however, there were some distinguishing features apart from the absence of an occluded mesenteric vessel in ischaemic entero- colitis and its presence in superior mesenteric artery thrombosis. In patients with ischaemic enterocolitis the lesions were distributed in a patchy manner in the affected gut; in patients with superior mesenteric artery thrombosis, the lesions were diffuse and confined to the distribution of that artery. The patchy nature of the lesions of ischaemic enterocolitis is illustrated in the case of a patient who had a normal jejunal biopsy but who was found at laparatomy to have ischaemic stricture and ulceration of a segment of jejunum. A second finding of help in distinguishing the two conditions is the presence of partial mucosal infarction in some patients with ischaemic enterocolitis. This was not observed in patients with superior mesenteric artery thrombosis in whom the full thickness of the mucosa was always infarcted.

The presence of haemosiderin-laden macrophages has been described as being of considerable help in the diagnosis of ischaemic enterocolitis (Marston et al, 1966; Morson, 1968). In none of the patients in the present series was haemosiderin seen. This may be due to the fact that most of the specimens were obtained during the acute phase of the disease. Fibrosis of the submucosa and muscularis propria in the patients with strictures suggested healing lesions, but no haemosiderin was found in them.

However, the general pattern of clinico-pathologe is the same as in the experience of others, and we have found that mucosal fibrosis has been of help in diagnosis. Ischaemic fibrosis is well recognized in many organs, eg, the kidney, as a progressive atrophy of parenchyma accompanied by its replacement with collagen. The same process appears to occur in the gut, with preferential involvement of the mucosa. The latter remains intact but there is an expansion of the lamina propria with eosinophilic cellular material which stains as collagen with van Gieson's method. The crypts are separated by this substance, and in the small intestine normal villi disappear and mucosal thickness is reduced. It is likely that these changes affect the mucosa first because it is furthest from the point of arterial supply, but it may well be that minor histological changes are more readily detected within the mucosa, and that similar degrees of change in submucosal collagen are undetectable. This mucosal fibrosis was not observed in the control patients who had no ischaemia of the bowel. The average age of the control patients $(55 \mathrm{yr})$ is less than that of the patients with ischaemia of the bowel $(66 \mathrm{yr})$. This was due to the inclusion in the former group of three patients in their third decade who had Crohn's disease. The occurrence of fibrosis in Crohn's disease was felt to justify their inclusion because of the possibility of mucosal fibrosis occurring in this condition. However none was seen. We feel that this mucosal change can be of help in biopsies, and that 
rectal or jejunal fibrosis of this kind should lead one to suggest ischaemia as a probable basis for symptoms. Its finding in the jejunum of a patient with superior mesenteric artery thrombosis supports the view that ischaemia is the basis for mucosal fibrosis in ischaemic enterocolitis.

\section{References}

Larsen, A. (1970). Non-occlusive intestinal gangrene.Acta chir. scand., 136, 227-234.

McGovern, V. J., and Goulston, S. J. M. (1965). Ischaemic enterocolitis. Gut, 6, 213-220.

Marston, A., Pheils, M. T., Thomas, M. L., and Morson, B. C. (1966). Ischaemic colitis. Gut, 7, 1-15.
Ming, S. C. (1965). Hemorrhagic necrosis of the gastrointestinal tract and its relation to cardiovascular status. Circulation, 32, 332-341.

Morson, B. C. (1966). Histopathology of ischaemic colitis. Proc. roy. Soc. Med., 59, 889-890.

Morson, B. C. (1968). Ischaemic colitis. Postgrad. med. J., 44, 665-666. Moulonguet, P. (1931). Bull. Soc. nat chir., 57, 1494, 1505 (Quoted by Larsen, A., 1970.)

Musa, B. U. (1965). Intestinal infarction without mesenteric vascular occlusion. A report of 31 cases. Ann. intern. Med., 63, 783-792.

Penner, A., and Bernheim, A. I. (1939). Acute postoperative enterocolitis. Arch. Path., 27, 966-983.

Wang, C. C., and Reeves, J. D. (1960). Mesenteric vascular disease. Amer. J. Roentgenol., 83, 895-908.

Wilson, R., and Qualheim, R. E. (1954). A for $n$ of a cute haemorrhagic enterocolitis afflicting chronically ill individuals. Gastroenterology, 27, 431-444. 